\title{
Hot Cross Bun Sign: A Case Report of Multiple System Atrophy Presenting Predominantly as Respiratory Insufficiency
}

\author{
Margiben Tusharbhai Bhatt, R. Sunil \\ Department of Critical Care Medicine, Kasturba Medical College, Manipal, Manipal Academy of Higher Education, Manipal, Karnataka, India
}

\section{Abstract}

Multiple system atrophy (MSA) was a multisystem disorder usually associated with Parkinsonism, MSA-p subtype or with cerebellar ataxia, and MSA-c subtype. Respiratory insufficiency can be a part of the clinical manifestation of multiple-system atrophy. It can sometimes present as primary respiratory failure, mild motor function disturbance, or autonomic dysfunction. In this article, we present a case of a 60-year-old lady who was admitted to the intensive care unit with fever and shortness of breath of 3 days duration with an initial working diagnosis of community-acquired pneumonia requiring invasive mechanical ventilation. She had a history of daytime sleepiness and gait disturbance. A thorough neurological evaluation was done to ascertain the cause of difficulty in weaning her off the ventilator. Magnetic resonance imaging of the brain revealed atrophy of bilateral superior cerebellar peduncles and pons with the "hot cross bun sign" suggestive of multiple system atrophy. Thus, a prompt neurological examination and work-up, including brain imaging, are mandated in dealing with such cases of difficult to wean patients.

Keywords: Hot cross bun sign, magnetic resonance imaging brain, multiple system atrophy, respiratory insufficiency

\section{INTRODUCTION}

Multiple system atrophy (MSA) is a rare neurodegenerative disorder. Typical presentation includes motor dysfunction, along with autonomic disturbances. Motor dysfunction can be predominantly Parkinsonism (p) type or a cerebellar (c) type. In addition, respiratory insufficiency, stridor, snoring, and central sleep apnea are also commonly seen in MSA. ${ }^{[1]}$ MSA is diagnosed on the basis of clinical criteria (which does not involve respiratory symptoms) supported by neuroimaging. ${ }^{[2]}$ Here, we present a case that initially presented with predominantly respiratory insufficiency. She was found to have a mild motor and autonomic problems. Later in the course of evaluating for the difficulty in weaning from the ventilator, she was diagnosed to have MSA based on neurological and radiological features.

\section{Case Report}

A 60-year-old female, homemaker, with nil premorbid conditions, presented to our hospital with chief complaints of fever and shortness of breath of 3 days duration. The patient, over the past 3 days, started developing fever which was high grade, intermittent, associated with difficulty in breathing and altered

\begin{tabular}{|l|l|}
\hline \multicolumn{2}{|c|}{ Access this article online } \\
\hline Quick Response Code: & Website: \\
\hline & www.ijrc.in \\
\cline { 2 - 2 } & \\
\hline
\end{tabular}

sensorium. On arrival at the hospital, she was hemodynamically stable but tachypneic with a respiratory rate of 40 breaths/ min. Her sensorium was E2V2M5, and arterial blood gases were suggestive of mild hypoxemia and respiratory acidosis. Endotracheal intubation and mechanical ventilation were initiated in view of altered sensorium and tachypnea. She was moved to a dedicated severe acute respiratory illness intensive care unit for further management in view of the ongoing COVID-19 pandemic, pending nasopharyngeal swab results.

Routine blood tests, including complete blood count, renal and liver function tests, serum electrolytes, blood culture, endotracheal sample culture, and nasopharyngeal swab for COVID-19, were sent. COVID-19 report turned out to be negative. Her baseline blood investigations were normal,

Address for correspondence: Dr. Margiben Tusharbhai Bhatt, Department of Critical Care Medicine, Kasturba Medical College and Hospitals, Manipal Academy of Higher Education, Manipal - 576 104, Karnataka, India. E-mail: drmargibhatt@hotmail.com

This is an open access journal, and articles are distributed under the terms of the Creative Commons Attribution-NonCommercial-ShareAlike 4.0 License, which allows others to remix, tweak, and build upon the work non-commercially, as long as appropriate credit is given and the new creations are licensed under the identical terms.

For reprints contact:WKHLRPMedknow_reprints@wolterskluwer.com

How to cite this article: Bhatt MT, Sunil R. Hot cross bun sign: A case report of Multiple System Atrophy presenting predominantly as respiratory insufficiency. Indian J Respir Care 2021;10:148-51.

Received: 29-06-2020

Accepted: $27-09-2020$ Published: $31-01-2021$ 
with no liver or renal dysfunction except for high white blood cell counts. Chest X-ray (CXR) was showing the right lower zone opacities, for which she was started on piperacillin-tazobactam [Figure 1]. Blood and endotracheal aspirate cultures were sterile. Gradually, her sensorium improved, and her CXR improved [Figure 2]. She was having 4-5 episodes of bradycardia and hypotension which responded to injection atropine $1 \mathrm{mg}$ intravenously. Twelve lead electrocardiograph and trans-thoracic echocardiography were done, which did not reveal any structural heart abnormality. These bradycardic episodes were also not related to medications, metabolic impairment, electrolytes imbalance, or procedures such as endotracheal suctioning and nursing care.

Even after the improvement of her lung condition and improvement in the mental status, there was a difficulty in weaning her off the ventilator. The A-airway/lung, B-Brain, C-Cardiac, D-Diaphragm, and E-Endocrine (ABCDE) of weaning failure, that is, airway/lung, brain, cardiac, diaphragm, and endocrine was assessed, and it was found that the patient was requiring higher pressure support to maintain adequate tidal volumes. A diaphragmatic excursion, which was done at the bedside by point of care ultrasound, was suggestive of bilateral decreased diaphragmatic excursion. The patient was having respiratory fatigue after $1-2 \mathrm{~h}$ of continuous spontaneous ventilation mode. On detailed history from the patient's attendant, it was found that the patient was having mild difficulty in walking for the past 3 years, but she was able to do household chores. She was also having generalized tiredness and daytime sleepiness for the past 2-3 years. On neurological examination, it was found that she was having bilateral upper limb power of 4/5. Reflexes were brisk, and bilateral plantar response was flexor. Cranial nerve examination was normal except for decreased gag reflex.

Magnetic resonance imaging (MRI) brain and cervical spine were done as per neurology advice. MRI cervical spine was suggestive of disc bulge with mild spinal canal stenosis and indentation on the spinal cord, but there was no evidence of nerve root compression at the level of C5-C6

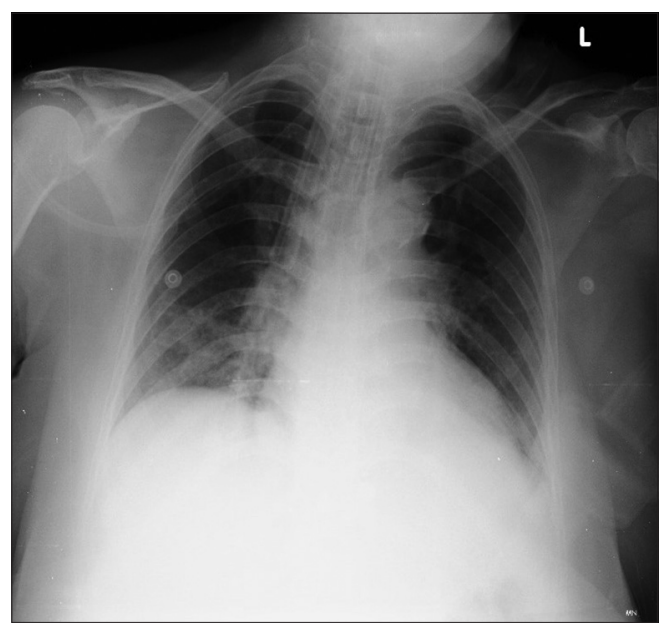

Figure 1: Chest X-ray on admission and $\mathrm{C} 6-\mathrm{C} 7$ vertebrae. Bilateral upper limb and lower limb nerve conduction studies were done, which were showing peripheral axonal motor neuropathy. MRI brain was suggestive of disproportionate atrophy of bilateral cerebellar hemispheres and brain stem with $\mathrm{T} 2$ hyperintensity forming a cross on axial images through the pons, the "hot cross bun (HCB) sign" and in the bilateral superior cerebellar peduncles (right $>$ left) suggestive of multiple system atrophy [Figures 3-5].

Multidisciplinary counseling involving internal medicine, critical care medicine, and neurology was done with family discussion on further goals of care, keeping multiple system atrophy in mind. A plan for elective tracheostomy was discussed owing to the expected prolonged duration of mechanical ventilation, but patient's family opined to move her to other medical facilities, and hence, she was discharged against medical advice.

\section{Discussion}

MSA is one of the rare neurodegenerative diseases. The term, multiple system atrophy, was coined to include three clinical syndromes, namely olivopontocerebellar atrophy (OPCA), Shy-Drager syndrome, and striatonigral degeneration. Striatonigral degeneration was subsequently named as MSA with primarily Parkinsonism type, whereas OPCA was later named as MSA with primarily cerebellar ataxia type. ${ }^{[3]}$ When autonomic dysfunction is seen predominantly, the disease is named as "Shy-Drager syndrome." The prevalence of MSA is approximately $2-5$ cases $/ 100,000$ population. ${ }^{[4]}$ The mean age at the onset is around $53(36-74)$ years. ${ }^{[5]}$

Loss of neuronal cells and gliosis spreading across the central nervous system is the central pathological process in MSA. Areas of the brain predominantly affected are the caudate nucleus, pontine nuclei, putamen, substantia nigra, inferior olivary nucleus, Purkinje cell layer of the cerebellum, and intermediolateral cell columns. Pathological hallmarks of MSA are argyrophilic cell inclusion bodies in oligodendrocytes in the regions involved in the central nervous system. ${ }^{[6]}$ Expected

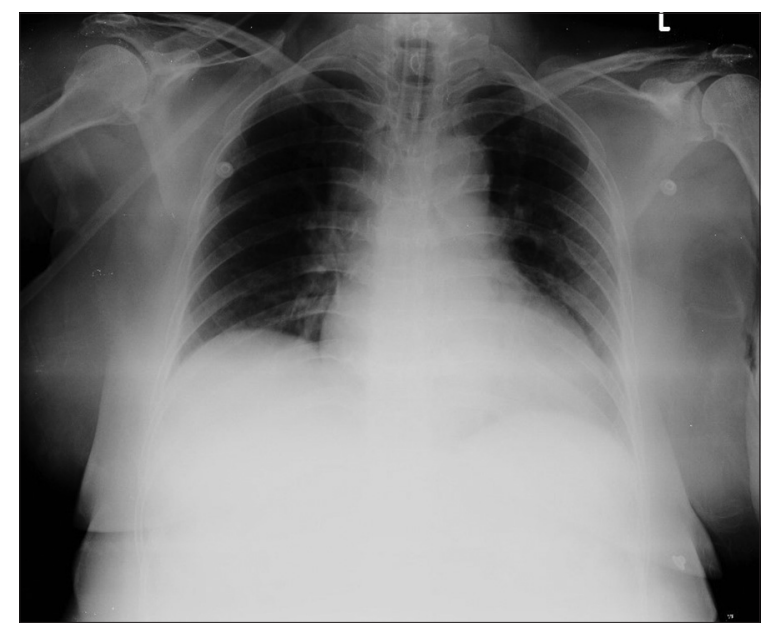

Figure 2: Improvement on chest X-ray 


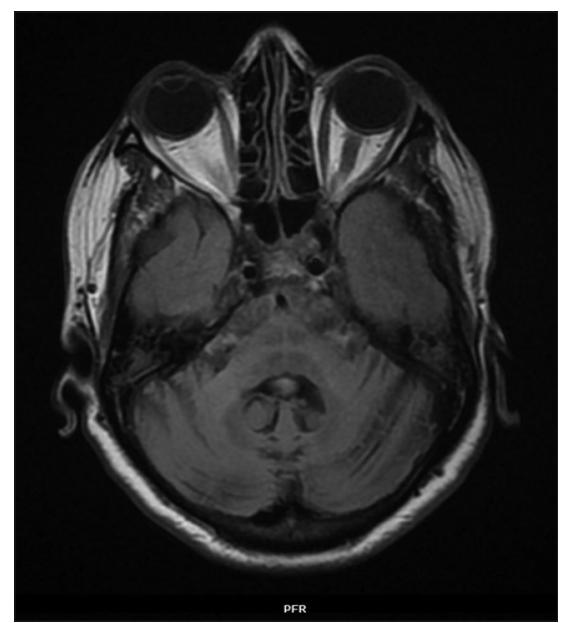

Figure 3: Magnetic resonance imaging brain - T2 flair image showing "hot cross bun sign"

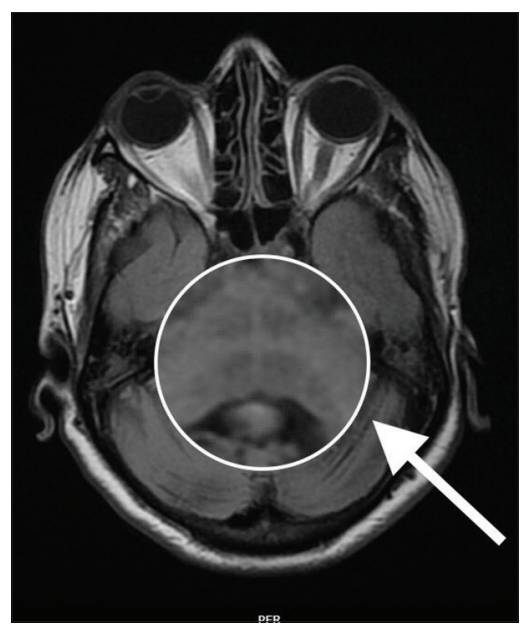

Figure 5: Magnetic resonance imaging brain - zoomed view of "hot cross bun sign"

survival from the onset of symptoms is $8-10$ years. It is still not clear what exactly causes mortality in the case of MSA. It has been observed that a large number of patients die during the night-time, probably because of respiratory insufficiency.

Patients with MSA are shown to have minimal chemosensitivity to hypoxia. They are at high risk of hypoxic respiratory failure with the failed compensatory mechanism. They often have respiratory rhythm disturbances during sleep. This can explain patients dying despite tracheostomy. ${ }^{[7,8]}$ As per the second consensus statement on MSA, diagnostic criteria for possible MSA are as follows: Parkinsonism symptoms or cerebellar symptoms, minimum of one feature indicating autonomic dysfunction, and at least one of the following additional criteria: MRI evidence of atrophy in the putamen, middle cerebellar peduncle, or pons and hypometabolism in putamen on positron emission tomography scan. One of the interesting radiological features of MSA is HCB sign on MRI brain. HCB sign is seen as the cross-pattern in the pontine area on MRI [Figures 3-5]. The name is derived from a sweet bun which has a cross at

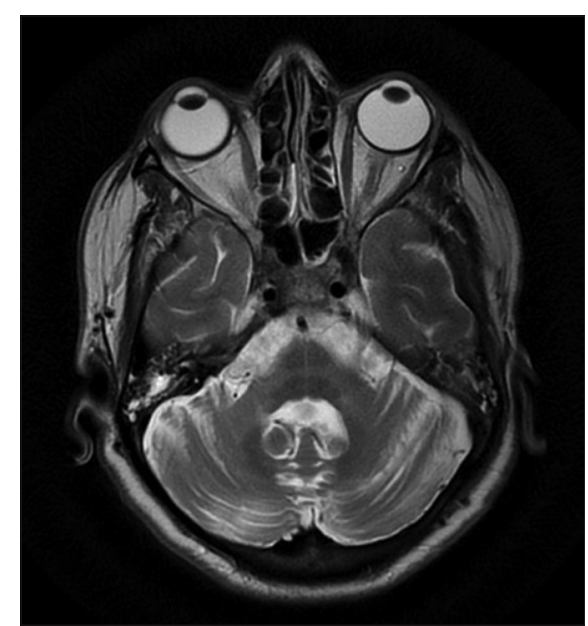

Figure 4: Magnetic resonance imaging brain T2 propeller image

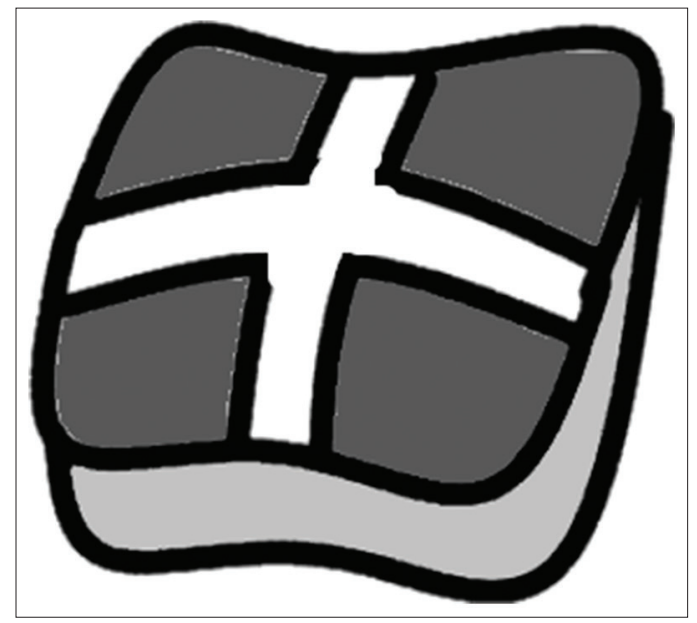

Figure 6: Pictorial depiction of hot cross bun

the top and eaten traditionally on Good Friday [Figure 6]. The $\mathrm{HCB}$ sign is due to selective loss of transverse pontocerebellar fibers without pontine tegmentum and corticospinal tracts being affected. Although first described in MSA individuals with the predominant cerebellar ataxia form, it is not pathognomonic of this disease.$^{[9,10]}$ In our case, the patient had fulfilled clinical and radiological criteria to establish a diagnosis of possible MSA. As no other cause of bradycardia was found in our patient and she was diagnosed to have possible MSA in which autonomic dysfunction is commonly seen, we attributed bradycardia as a part of MSA presentation.

Our patient had difficulty in weaning from the ventilator. The ABCDE approach ${ }^{[11]}$ is one of the structured approaches to look for the cause of weaning failure. ABCDE the above mentioned factors such as airway resistance and compliance, lung pathology, cardiac abnormality, endocrine, or metabolic causes were evaluated and ruled out as a cause of weaning failure. The neurologic evaluation followed by neuroimaging and point of care ultrasound bedside revealed decreased gag reflex, HCB sign on MRI, and bilateral decreased diaphragmatic excursion, respectively. 
Respiratory insufficiency is one of the rare manifestations of MSA. There are very few articles in the literature suggesting the same. ${ }^{[12-14]}$ Glass et al. ${ }^{[14]}$ reported a case series of total of six cases of MSA having respiration disturbances in an early stage of the disease in 2006. They found that respiratory problems had overshadowed the neurologic complaints in all of these cases. On initial presentation, MSA was not suspected, which was diagnosed later in the course. They also found that respiratory rhythm disturbance was the main culprit for the patients dying despite tracheostomy. In patients with obstructive sleep apnea, cardiac rhythm disturbances and even sinus arrest also were noted. This explains why the disease has an overall bad prognosis. When dealing with the cases of progressive alveolar hypoventilation, difficulty in weaning from the ventilator, or respiratory failure, it is important to keep MSA in mind as a differential diagnosis. This case report emphasizes the importance of the same.

\section{Conclusion}

MSA is a progressive disease with a poor prognosis. Clinical information is sometimes remained unclear and specific diagnostic modalities are required to complete the diagnosis. Sometimes, the primary manifestation of the disease is respiratory insufficiency, with mild motor and autonomic dysfunction. In such cases, while evaluating the causes for difficulty in weaning from the ventilator or unexplained respiratory distress, MSA should be kept in mind.

\section{Financial support and sponsorship}

Nil.

\section{Conflicts of interest}

There are no conflicts of interest.

\section{RefEREnCES}

1. Sadaoka T, Kakitsuba N, Fujiwara Y, Kanai R, Takahashi H. Sleep-related breathing disorders in patients with multiple system atrophy and vocal fold palsy. Sleep 1996;19:479-84.

2. Gilman S, Wenning GK, Low PA, Brooks DJ, Mathias CJ, Trojanowski JQ, et al. Second consensus statement on the diagnosis of multiple system atrophy. Neurology 2008;71:670-6.

3. Finke C, Siebert E, Plotkin M, Wenning GK, Reuter U. Multiple system atrophy masking multiple sclerosis. Clin Neurol Neurosurg 2010;112:59-61.

4. Tison F, Yekhlef F, Chrysostome V, Sourgen C. Prevalence of multiple system atrophy. Lancet 2000;355:495-6.

5. Swan L, Dupont J. Multiple system atrophy. Phys Ther 1999;79:488-94.

6. Lantos PL. The definition of multiple system atrophy: A review of recent developments. J Neuropathol Exp Neurol 1998;57:1099-111.

7. Wenning GK, Ben Shlomo Y, Magalhães M, Daniel SE, Quinn NP. Clinical features and natural history of multiple system atrophy. An analysis of 100 cases. Brain 1994;117:835-45.

8. Silber MH, Levine S. Stridor and death in multiple system atrophy. Mov Disord 2000;15:699-704.

9. Stefanova N, Bücke P, Duerr S, Wenning GK. Multiple system atrophy: An update. Lancet Neurol 2009;8:1172-78.

10. Way C, Pettersson D, Hiller A. The 'hot cross bun' sign is not always multiple System atrophy: Etiologies of 11 cases. J Mov Disord 2019;12:27-30.

11. Heunks LM, van der Hoeven JG. Clinical review: The ABC of weaning failure--a structured approach. Crit Care 2010;14:245.

12. Cormican LJ, Higgins S, Davidson AC, Howard R, Williams AJ. Multiple system atrophy presenting as central sleep apnoea. Eur Respir J 2004;24:323-5.

13. Lim YS, Kennedy NJ. Multiple system atrophy as a cause of upper airway obstruction. Anaesthesia 2007;62:1179-82.

14. Glass GA, Josephs KA, Ahlskog JE. Respiratory insufficiency as the primary presenting symptom of multiple-system atrophy. Arch Neurol 2006;63:978-81. 